\title{
OPTIMIZATION OF PARAMETERS OF HEATING ELEMENTS FOR FLOOR PANEL OF PIGLETS RESTING PLACES
}

\author{
V.Zagorska ${ }^{1}$, U.Iljins ${ }^{2}$ \\ 1- Latvia University of Agriculture, Institute of Mechanics \\ J.Čakstes bulv. 5, Jelgava, LV 3001, Latvia \\ Ph.: +(371)29740492, e-mail: vzagorska@gmail.com \\ 2- Latvia University of Agriculture, Department of Physics
}

\begin{abstract}
The article deals with problem solving of mathematical physics using the method of separation of variables optimizing heating element - optimizing water tube parameters (tube material, radius, insulation thickness, choosing appropriate surrounding environment). For ensuring piglets comfort, concrete floor panels heated by electric current or hot water are used. If an electro-heated cable in the panels body is placed, than amount of heat conducted from the cable is the same along all the length of the cable. If hot water circulating through tube is used, than amount of heat energy taken off the heater decreases along its length. The aim of the research is to create the mathematical model of a water tube, were water temperature is gradually decreasing. This model is needed to make precise calculations of the heating panel for piglets, to ensure equal temperature distribution over the upper surface of the panel, taking into account mathematically calculated temperature decrease of the heat source.
\end{abstract}

Keywords: mathematical modeling, heated floors, water tube.

\section{Introduction}

New born piglets together with the sow are kept. The optimal surrounding air temperature for a sow is about $16 \ldots 20^{\circ} \mathrm{C}$, but for new born piglet during the first days of their life the temperature in its lairs has to be within the limits of $32 \ldots 36^{\circ} \mathrm{C}$. Gradually the lair's temperature must be decreased until $22 \ldots 24^{\circ} \mathrm{C}$ when piglets are two months of age and weaned [Priekulis et al., 1992]. That means that comfortable surrounding temperature for sows and piglets is different despite the fact that during first days they are kept together. Therefore in cold winter countries like Latvia piglets resting place local warming ought to be used.

For local warming heating panels are usually used. They can be made from different materials and using different production technologies. The energetic efficiency of the panels will result from the integrated insulation which minimizes the downward heat emission and from the even distribution over the panel. To improve evenness of the temperature distribution it is necessary to make precise mathematical model of the heating panel and then to check it experimentally. Nowadays very popular are plastic pads which are filled in with water, but the evenness of the temperature is not so good [MIK, 2009], as it can be achieved using tubes with hot water placed into some kind of solid material, in our examples we used data for concrete, but there is a possibility to use another materials (with lesser abrasive properties) [Zoric. M. et al., 2009]. The energy consumption of the calculated concrete panel is lower than the most popular panels in the market is as well, comparing $320 \mathrm{~W} \mathrm{~m}^{-2}$ [MIK, 2009], to $290 \mathrm{~W} \mathrm{~m}^{-2}$ at the same working temperatures. In terms of energy cost this leads to the enormous energy economy and decrease of $\mathrm{CO}_{2}$ pollutions as well. 


\section{Materials and methods}

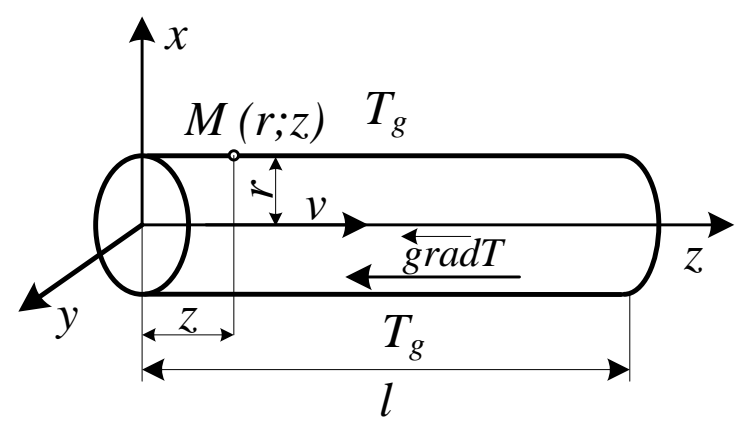

\section{Fig.1. Pipe element for calculation of temperature drop over the fluid flow in the tube}

Assuming, that tube is being bathed by environment, which temperature $T_{0}$ and flowing into the tube fluid temperature $\mathrm{T}_{1}>\mathrm{T}_{0}$ (the fluid gives the heat energy to he surrpunding environment). These processes can be described with equation (1) [Riekstins, 1969]:

$$
a \Delta T-\overrightarrow{v g r a d} T=0 .
$$

For concrete problem formulation it is necessary to give boundary conditions. According to our task, the fluid flows into the tube with temperature $\mathrm{T}_{1}(2)$ :

$$
T \|_{z=0}=T_{1} .
$$

Conditionally we can accept that outflow fluid temperature is equal to surrounding air temperature (3):

$$
T \|_{z \rightarrow \infty}=T_{0}
$$

It is necessary to formulate the third type boundary conditions as well for the tube surface (4).

$$
-\left.\lambda \frac{\partial T}{\partial r}\right|_{r=R}=\alpha\left(T \|_{r=R}-T_{0}\right),
$$

where

$$
\alpha \text { - heat transfer coefficient, } \mathrm{W} \mathrm{m}{ }^{-2} \mathrm{~K}^{-1} \text {; }
$$

$\lambda_{2}$ - thermal conductivity, $\mathrm{W}(\mathrm{m} \mathrm{K})^{-1}$.

Now we have defined the problem of mathematical physics, which contains equation (1) and border conditions (2-4). For solving such a problem, we will use the method of separation of variables. In cylindrical coordinate system the Laplace operator is written in a form:

$$
\Delta=\frac{1}{r} \frac{\partial}{\partial r}\left(r \frac{\partial}{\partial r}\right)+\frac{\partial^{2}}{\partial x^{2}}=0 .
$$

So, equation (6) is a stationary equation, which contains conditions of fluid flow:

$$
\frac{1}{r} \frac{\partial}{\partial r}\left(r \frac{\partial T}{\partial r}\right)-\frac{\vec{y}}{a} \frac{\partial T}{\partial z}+\frac{\partial^{2} T}{\partial z^{2}}=0
$$

The solution of the problem we will look for as sum of endless row (7):

$$
T\left(r_{s} z\right)=T_{0}+\sum_{k} R_{k}(r) \cdot Z_{k}(r) .
$$


Inserting representation (7) into the basic equation (6), accordingly substituting $\frac{\partial R_{k}}{\partial r}=R_{k}^{\prime}$ and $\frac{\partial Z_{k}}{\partial z}=Z_{k}^{\prime}$.we will get the following:

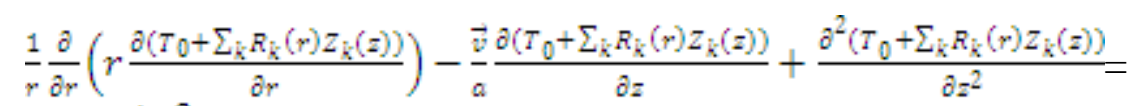

$$
\begin{aligned}
& =\frac{1}{r} \frac{\partial}{\partial r} \sum_{k}\left(r \cdot\left(R_{k}{ }^{b} \cdot Z_{k}\right)\right)^{b}-\sum_{k} \frac{v}{a} R_{k} Z_{k}{ }^{b}+\sum_{k} R_{k} Z_{k}{ }^{v}= \\
& \sum_{k} \frac{1}{r} Z_{k}\left(R_{k}{ }^{b}+r R_{k}{ }^{w}\right)-\frac{v}{a} R_{k} Z_{k}{ }^{b}+R_{k} Z_{k}{ }^{n}=0
\end{aligned}
$$

The solution of the equation (8) is found only in case, when each member of the sum row is equal with zero:

$$
\frac{1}{r} Z_{k}\left(R_{k}^{*}+r R_{k}^{s f}\right)-\frac{v}{a} R_{k} Z_{k}^{*}+R_{k} Z_{k}^{s p}=0
$$

Now, dividing equation (9) with $R_{k} Z_{k}$, we can get equation were the right side is dependant from one argument, and the left side from another argument (10):

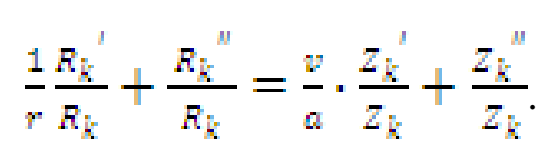

To get 2 equations with one unknown, we need to compare it with free chosen constant $\mu_{k}^{2}$ (11):

$$
\left\{\begin{array}{l}
\frac{1}{T} \frac{R_{k}{ }^{p}}{R_{k}}+\frac{R_{k}{ }^{s p}}{R_{k}}=\mu_{k s}^{2} \\
\frac{v}{a} \cdot \frac{Z_{k}}{Z_{k}}+\frac{Z_{k}}{Z_{k}}=\mu_{k}^{2}
\end{array}\right.
$$

After algebraic changes we obtain two second order differential equations (12):

$$
\left\{\begin{array}{l}
r R_{k}{ }^{s f}+R_{k}+\mu_{k}^{2} r R_{k}=0 \\
Z_{k}+\frac{v}{a} \cdot Z_{k}+\mu_{k}^{2} Z_{k}=0
\end{array}\right.
$$

The first system's (12a) equation is special case of the general equation (13) which is called Bessel equation:

$$
r^{2} R_{k}^{\prime \prime}+r R_{k}^{\prime} \pm\left(\mu_{k}^{2} r^{2}-v^{2}\right) R_{k}=0,
$$

where $v$ is numeral parmeter, in our case $v=0$.

The solution of the Bessel equation is expressed with Bessel functions $J_{v}\left(\mu_{k} r\right)$. According to it the solution of the equation (12a) is:

$$
\begin{gathered}
R_{k}(r)=A_{k} \cdot J_{0}\left(\mu_{k} r\right)+B_{k} \cdot Y_{0}\left(\mu_{k} r\right), \\
Y_{0}=0 \Longrightarrow B_{k} \cdot\left(\mu_{k} r\right)=0, \\
R_{k}(r)=A_{k} \cdot J_{0}\left(\mu_{k} r\right),
\end{gathered}
$$

where $A_{k}$ is free chosen integration constant;

$I_{0}\left(\mu_{k} r\right)$ - Bessel function, when parameter $v=0$. 
The equation (12b) is the 2 nd order differential equation with constant coefficients, dividing it with $Z_{k}$, and making substitution $\frac{\text { wep }}{\lambda}=2 \beta$ it is written in the following form (17):

$$
Z_{k}{ }^{s s}(z)-2 \beta Z_{k}(z)-\mu_{k}^{2} Z_{k}(z)=0 .
$$

The general solution of the equation (17) is:

$$
Z_{k}(z)=C_{k} e^{E_{1} z}+D_{k} e^{E_{2} z}
$$

where $C_{k}$ and $D_{k}$ are free chosen integration constants,

$\xi$ - particular value.

$$
\begin{aligned}
& \xi_{1}=\beta+\sqrt{\beta^{2}+\mu_{k}^{2}} \\
& \xi_{2}=\beta-\sqrt{\beta^{2}+\mu_{k}^{2}}
\end{aligned}
$$

Further process for solution finding is being made: the expressions (18) and (16) are inserted into the equation (7), the equation (21) is obtained. The equation (21) is being inserted into the boundary conditions $(2,3)$ in consecutive order. To satisfy boundary conditions (2), no restrictions are introduced. The boundary conditions (3) can be satisfied, only when $z \rightarrow \infty$, then $e^{\left(\beta+\sqrt{\left.\beta^{2}+\mu_{k}^{x}\right) z}\right.} \rightarrow \infty$, it means, that we have to assume $C_{k}=0$, to satisfy boundary conditions (3). The following is obtained:

$$
T(r ; z)=T_{0}+\sum_{k} J_{0}\left(\mu_{k} r\right)\left(D_{k} e^{\left(\beta-\sqrt{\beta^{2}+\mu_{k}^{2}}\right) z}\right),
$$

where $-D_{k}$ is free chosen constant $A_{k} \cdot D_{k}$

As we see from equation (21) we do not know free chosen constants $\mu_{k}$ and $D_{k}$.

To determine free chosen constants $\mu_{k}$ we will use side surface boundary conditions (4), the equation (21) is inserted into the boundary conditions (4):

$$
\begin{gathered}
-\lambda \sum_{k} D_{k}\left[-J_{1}\left(\mu_{k} R\right)\right] \cdot \mu_{k} \cdot e^{\left(\beta-\sqrt{\beta^{2}+\mu_{k}^{2}}\right) z}= \\
\left.\alpha\left(T_{0}+\sum_{k} D_{k} \cdot J_{0}\left(\mu_{k} R\right)\right] \cdot e^{\left(\beta-\sqrt{\beta^{2}+\mu_{k}^{2}}\right) z}-T_{0}\right) .
\end{gathered}
$$

After row of algebraically changes we obtain:

$$
\mu_{k}=\frac{a}{2} \cdot \frac{d_{0}\left(\mu_{2} R\right)}{d_{1}\left(\mu_{2} R\right)}
$$

As Bessel function argument is dimensionless, than $\left[\mu_{k}\right]=\left[\mathrm{m}^{-1}\right]_{z}$ to introduce into the solution Biot number $\frac{\alpha}{\lambda} \cdot R=b$, we need to multiply equation (23) with $\mathrm{R}$ :

$$
\mu_{k} R=\frac{a}{2} \cdot R \cdot \frac{\mathbb{N}_{0}\left(\mu_{k} R\right)}{J_{1}\left(\mu_{R} R\right)}
$$

We will mark $\mu_{k} R$ as $X_{k}$ :

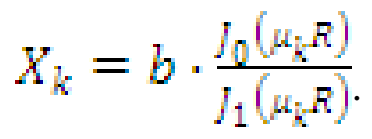

So we have got the transcendental equation for particular value determination. 
Now it is needed to determine $D_{k}$, to obtain it we will put general equation (21) into the boundary conditions (2), when $z \rightarrow 0$ :

$$
\begin{gathered}
T(r ; 0)=T_{1}=T_{0}+\sum_{k_{0}} J_{0}\left(\mu_{k} r\right) \cdot D_{k} e^{\left(\beta-\sqrt{\beta^{2}+\mu_{k}^{2}}\right)}= \\
=T_{0}+D_{1} J_{0}\left(\mu_{1} r\right)+D_{2} J_{0}\left(\mu_{2} r\right)+D_{3} J_{0}\left(\mu_{3} r\right)+\cdots+D_{k} J_{0}\left(\mu_{k} r\right) .
\end{gathered}
$$

Using properties of the particular functions' scalar multiplication multiplying with $r J_{0}\left(\mu_{k} r\right)$ and integrating, we obtain (27):

$$
\int_{0}^{R} r J_{0}\left(\mu_{k} R\right)\left(T_{1}-T_{0}\right) d r=D_{k} \int_{0}^{R} r J_{0}^{2}\left(\mu_{k} r\right)^{2} d r .
$$

The equation above enables to get solution for $D_{k}$ as division of two integrals:

$$
\left(T_{1}-T_{0}\right) \frac{d_{0}^{x} \gamma J_{0}\left(\mu_{k} x\right) d x}{\int_{0}^{2} x J_{0}^{2}\left(\mu_{k} x\right)^{2} d x}=D_{k} \text {. }
$$

Now we need to develop function into a row (29), where trigonometrically functions are taken for Furje rows:

where $-C_{k}$ is Furje coefficient.

$$
1=\sum_{k} C_{k} J_{0}\left(\mu_{k} r\right)
$$

From equation (28) it is seen, that:

$$
\begin{gathered}
D_{k}=\left(T_{1}-T_{0}\right) C_{k} ; \\
f(r)=\sum_{k} C_{k} J_{0}\left(\mu_{k} r\right),
\end{gathered}
$$

where $f(r)$ - whatever function, which can be impulse function as well.

Now we need both equations (29 and 30) to multiply with $r J_{0}\left(\mu_{k} r\right)$ and integrate from " 0 " to "R".

The particular function $I_{k}$ is obtained:

$$
I_{k}=\frac{R^{2}}{2}\left(J_{0}^{2}\left(\mu_{k} R\right)+J_{1}^{2}\left(\mu_{k} R\right)\right) \text {. }
$$

and

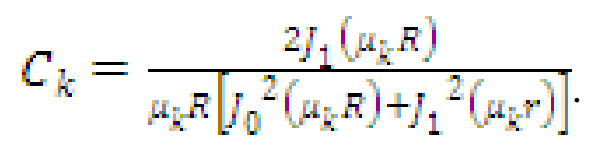

\section{Results and discussion}

The solution for determination the temperature drop during the fluid flow through the tube is obtained:

$$
T(r ; z)=T_{0}+2\left(T_{1}-T_{0}\right) \sum_{k=1}^{\infty} \frac{J_{1}\left(\mu_{k} R\right) \cdot J_{0}\left(\mu_{k} R\right) \cdot e^{\left(\beta-\sqrt{\beta^{2}+\mu_{k}^{2}}\right) z}}{\left(\mu_{k} R\right)\left[J_{0}{ }^{2}\left(\mu_{k} R\right)+J_{1}{ }^{2}\left(\mu_{k} R\right)\right]} .
$$

According to this equation, it is possible to calculate temperature drop during fluid flow through the tube at the different conditions. In the figure 1, different cases of the fluid flow are presented, three curves are being compared, when diameter of the tube and heat transfer coefficient is changeable, and other parameters remain constant.

From the picture it is seen, that temperature decrease of the fluid (in our case water) is exponentially dependant from the tube length. Three cases for simulation were chosen (diameter value $1 \mathrm{~cm}, 1.5 \mathrm{~cm}, 2 \mathrm{~cm}$ ). In the first case temperature drop during the $1 \mathrm{~m}$ fluid flow through the tube is $1.01 \%$, in the second and third cases $0.67 \%$ and $0.50 \%$ respectively. The value of the heat transfer coefficient was changed theoretically, but further it is necessary to make train of experiments to determine heat transfer coefficient dependency on tube 
diameter and other important parameter as flow speed, fluid type and surrounding environment.

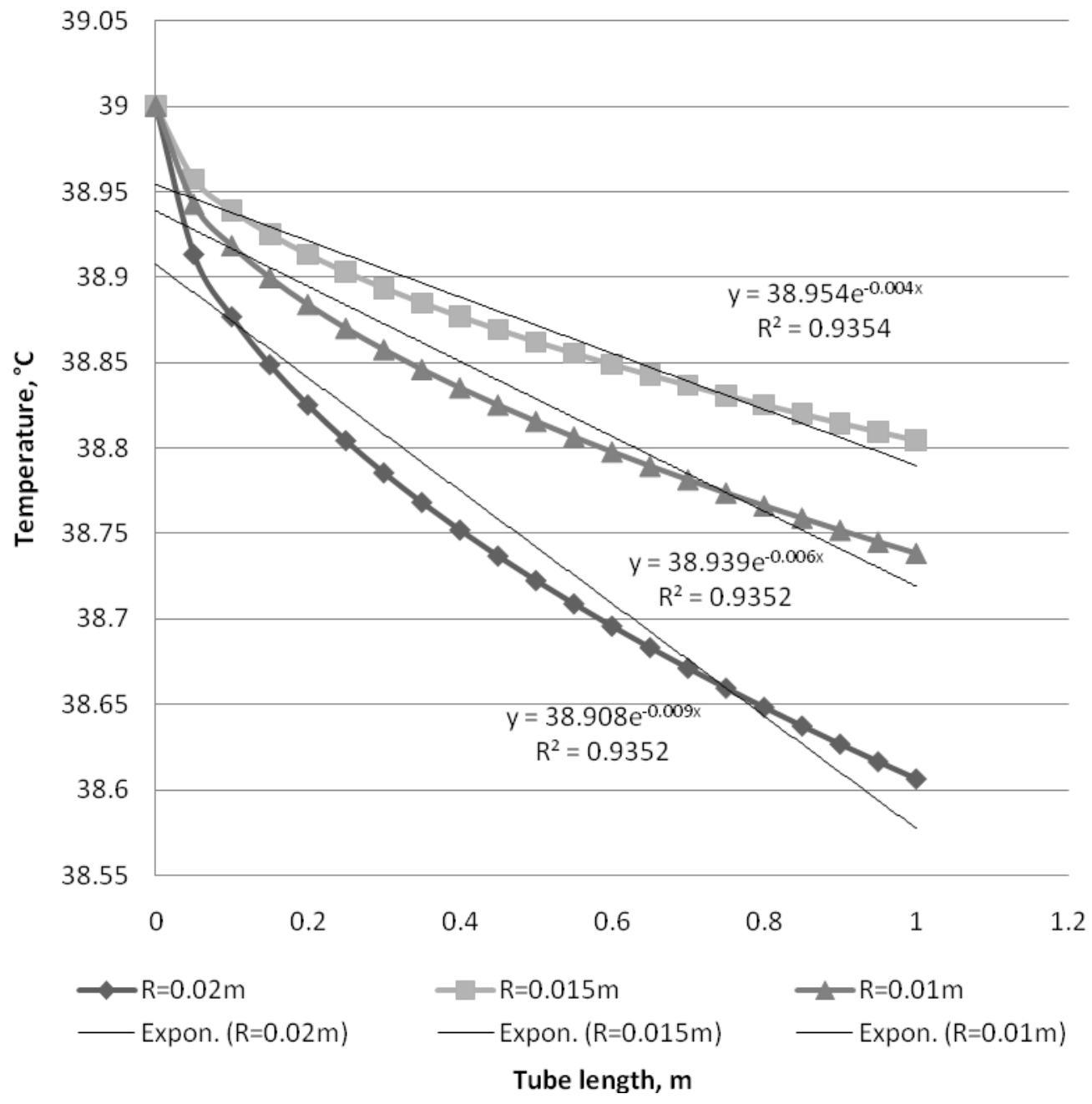

Fig. 1. Fluid flow through the water tube with different diameters

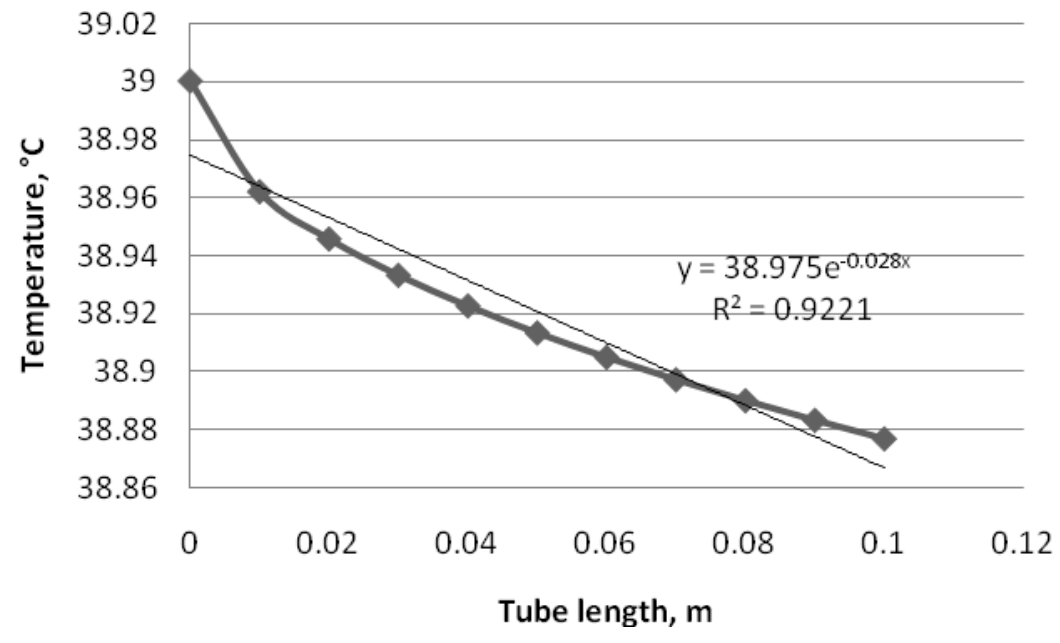

Fig. 2. Temperature drop of the fluid during the first $10 \mathrm{~cm}$ flow 


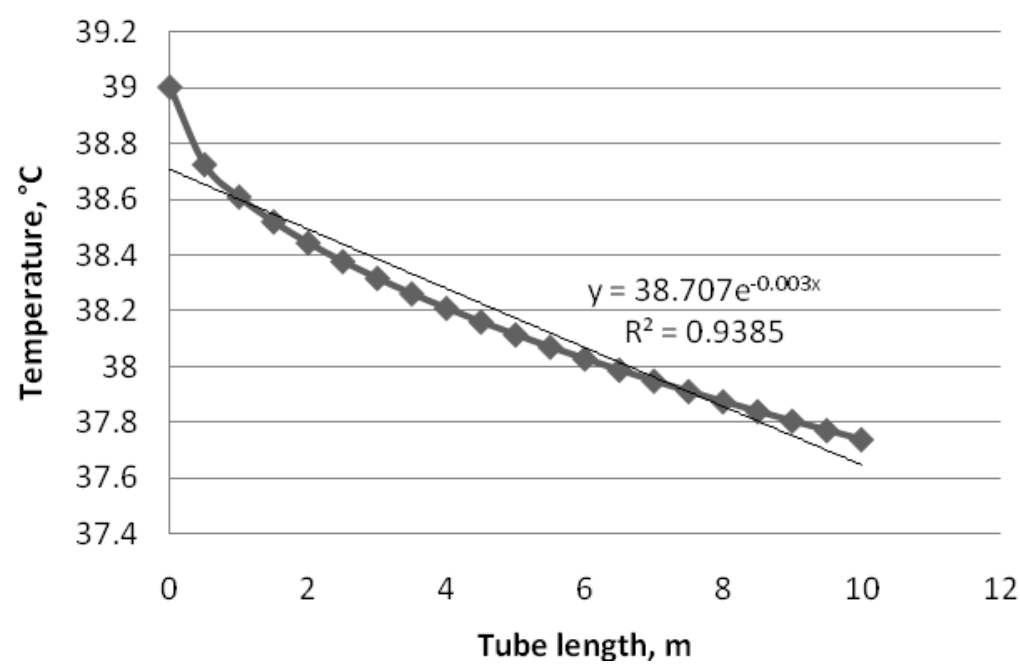

Fig. 3. Temperature drop of the fluid during the $10 \mathrm{~m}$ flow

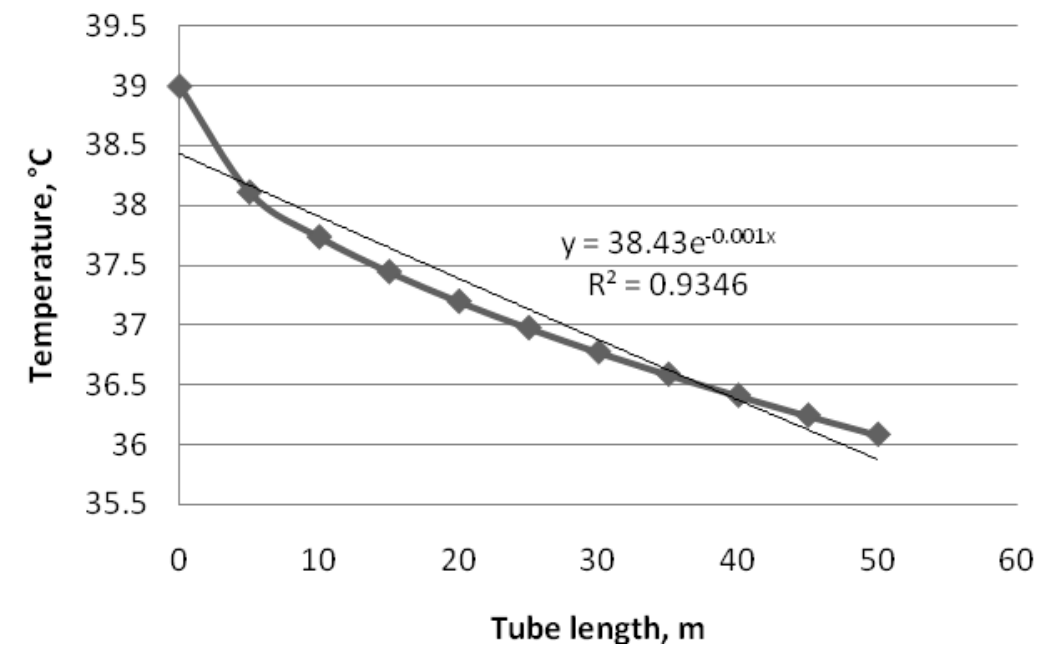

Fig. 4. Temperature drop of the fluid during the $50 \mathrm{~m}$ flow

Comparing figures two, three and four. We can see the tendency of the temperature drop depending on the fluid flow distance, for example, when fluid flows $10 \mathrm{~cm}$ the temperature drop of $0.32 \%$ occurs, but when distance is ten times greater, the temperature drop only for $1.01 \%$ appears, when we speak about longer distances, then at $50 \mathrm{~m}$ distance it will be $3.24 \%$. Using obtained formula it is possible to calculate the temperature drop of fluid at different fluid flow distances. Using previously obtained formulas [Zagorska V., et al. 2010], it is possible to make the model of the heating panel for various materials, flow rates, surrounding environment parameter values.

\section{Conclusions}

1. Formulas (33), (34) and (25) make the mathematical model of a tube with fluid flowing inside it at boundary conditions (2) to (4).

2. They enable to calculate the temperature drop, during the fluid flow at certain values of the parameters included into the formulas.

3. These calculations enable to analyze coherence among different parameters of a water tube. 
4. It is necessary to determine heat transfer coefficient dependence on the tube diameter, fluid flow speed and surrounding environment experimentally, using well known equations.

\section{Acknowledgment}

Paper becomes written by financial support of European Structural Fund - Attraction of human resources for investigation of renewable energy resources - realized by Project Department of Latvia University of Agriculture (contract no. 2009/0225/1DP/1.1.1.2.0/09/ APIA/VIAA/129).

\section{References}

1. Priekulis, J., Tilaks, S., Ziemelis, I. Mechanization of livestock farming. Zvaigzne, 1992, 379pp. (in Latvian)

2. MIK International AG. Technical report no. 7134 8988. Frankfurt, 2009, 18 pp. Available at: http://download.mik-online.de/agrar/en/news/thermow_TUEV_english.

3. Zoric, M., Nilsson, E. Incidence of lameness and abrasions in piglets in identical farrowing pens four different types of floor. Acta Veterinaria Scandinavica, 2009, 9pp. Available at: http://www.ncbi.nlm.nih.gov/pubmed/19463187

4. Riekstins, E.Methods of mathematical physics. Trans. Zvaigzne. 1969, p. 623. (in Latvian)

5. Zagorska V., Iljins U., Ziemelis I. Calculation of a Heated Floor Panel for Piglets Resting Places. Proceedings of the $4^{\text {th }}$ International Conference Trends in Agricultural Engineering 2010. Prague, Czech Republic, 2010, pp. 648-653. 